\title{
Diverse presentation of connective tissue disorders in pregnancy
}

\author{
Mini Bedi ${ }^{1}$, Sandeep Kaur ${ }^{1 *}$, Prashant Patil ${ }^{1}$, Kamaljeet Kaur ${ }^{2}$, \\ Harbhajan Kaur ${ }^{1}$, Harkiran Kaur ${ }^{3}$
}

${ }^{1}$ Department of Obstetrics and Gynecology, ${ }^{2}$ Department of Medicine, ${ }^{3}$ Dean of Faculty, AIMSR, Bathinda, Punjab, India

Received: 01 February 2021

Accepted: 06 March 2021

\section{*Correspondence:}

Dr. Sandeep Kaur,

E-mail: sandeepkang089@gmail.com

Copyright: (C) the author(s), publisher and licensee Medip Academy. This is an open-access article distributed under the terms of the Creative Commons Attribution Non-Commercial License, which permits unrestricted non-commercial use, distribution, and reproduction in any medium, provided the original work is properly cited.

\begin{abstract}
Connective tissue disorders (CTD) include a variety of chronic multisystem disorders including autoimmune conditions. Many of these conditions affect women of childbearing age and therefore pregnancy poses an important challenge for the caregivers. The precise knowledge of therapeutic safety and the effect of disease on pregnancy and vice versa are important to achieve best outcome. Hence, it is imperative to have a vast knowledge of disease with proper preconception counselling. We report series of cases of connective tissue disorders in pregnancy: tuberous sclerosis (TS), systemic lupus erythmatosis (SLE) and neurofibromatosis (NF) type 1. The first case with tuberous sclerosis was associated with obstetric complications. In both cases of SLE, we observed preterm delivery and IUGR. However, the course of SLE remained the same in both the cases. The case with NF 1 taught us that a normal obstetric outcome could be expected in pregnant women.
\end{abstract}

Keywords: Tuberous sclerosis, Neurofibromatosis, SLE, Connective tissue disorders, Pregnancy

\section{INTRODUCTION}

Tuberous sclerosis or Bourneville's disease is an autosomal dominant neurocutaneous disorder affecting multiple organs. It is characterized by presence of potato like tumours (tubers) in multiple organs. Classical Vogt's triad comprising of epilepsy, mental retardation and adenoma sebaceum is seen in $30 \%$ of cases. ${ }^{1}$ The case in this series is unique owing to the presence of all major criteria (of TS) in a single patient with occurrence of type 2 AV block during delivery.

Systemic lupus erythematosus (SLE) is an autoimmune condition that has multi-organ involvement. It is approximately ten times more common in women than in men and is often diagnosed during the childbearing years. SLE is known to increase the risk of spontaneous miscarriage; it can also cause fetal growth restriction and increased incidence of sudden intrauterine death, preeclampsia and preterm delivery. Management of women with lupus nephritis can be difficult, as the disease may mimic and overlap significantly with pre-eclampsia. Multidisciplinary management of pregnant women with SLE ensures optimal surveillance of both mother and fetus. $^{2}$

Neurofibromatosis type-1 (NF-1) is an autosomal dominant disorder with variable clinical manifestations such as cafe-an-lait spots, axillary freckling, cutaneous neurofibromas and iris hamartomas (Lisch nodules). It is characterized by different types of mutations of the NF-1 gene. ${ }^{3}$ The reported incidence of neurofibromatosis (NF) in pregnancy varies from 1:5000 to $1: 18500 .^{4}$

NF-1 is associated with fetal complications such as spontaneous miscarriage, preterm delivery, intrauterine growth retardation and stillbirths with maternal disease aggravation (hypertensive and cerebrovascular complications). ${ }^{5}$ 


\section{CASE SERIES}

\section{Case 1}

A 27 years old female presented to the emergency at 39 weeks of pregnancy in labour with fetal echocardiography showing rhabdomyomas and left ventricular outflow obstruction. She was examined and found to have shagreen patch (Figure 1), adenoma sebaceum (Figure 2), ash-leaf macules, periungual and subungual fibromas. USG abdomen revealed bilateral renal angiomyolipomas, hepatic angiomyolipomas with uterine myolipoma. Magnetic resonance imaging (MRI) brain showed cortical tubers in bilateral cerebral hemispheres with multiple subependymal nodules with subependymal giant cell astrocytoma left ventricle. During her delivery, she had cardiac arrest for which CPR was done and she was revived. Later type $2 \mathrm{AV}$ block was revealed in electrocardiography (ECG) and echocardiography revealed global hypokinesia of LV (ejection fraction 44\%). Patient has mild mental retardation. After delivery patient had bleeding per vaginum. Ultrasonography (USG) pelvis suggestive of differential diagnosis of gestational trophoblastic disease, RPOC and uterine angiomyolipomas for which chemotherapy with injection methotrexate was given.

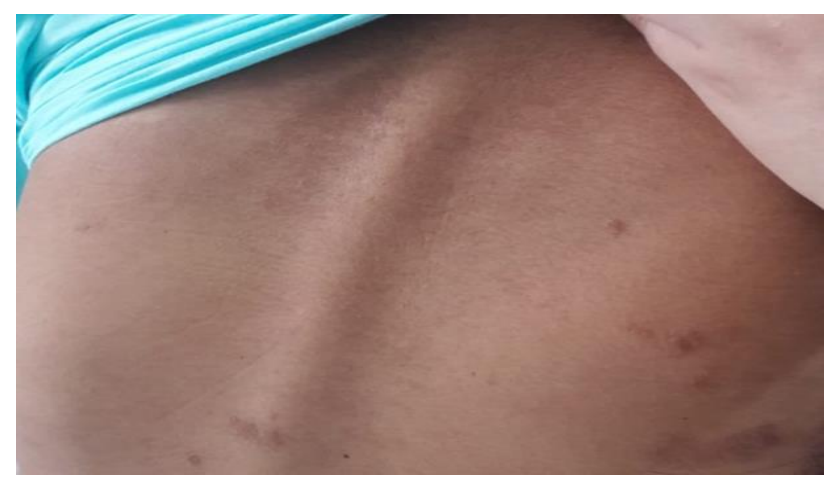

Figure 1: Shagreen patch.

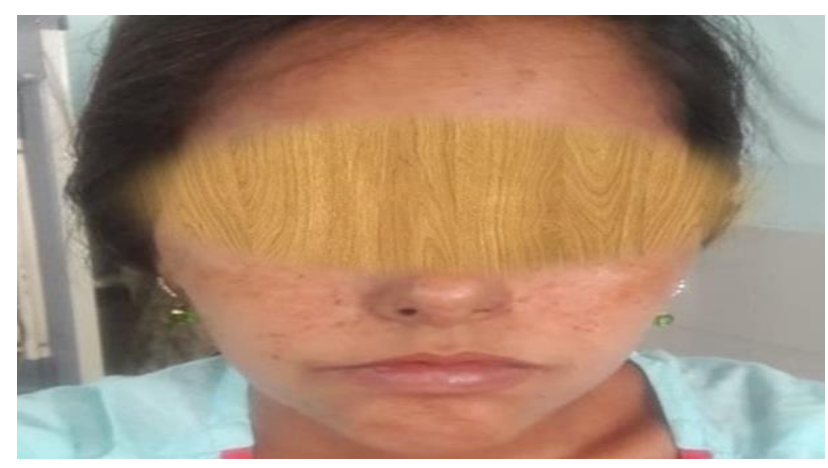

Figure 2: Adenoma sebaceum.

\section{Case 2}

A 34 years old female G2A1 with 32 weeks 4 days POG came to emergency with 8 months amenorrhea with high
$\mathrm{BP}$, headache and epigastric pain. She was referred as a case of IUGR, severe preeclampsia and SLE. On examination, blood pressure (BP) was 170/110 $\mathrm{mm} \mathrm{Hg}$, pulse rate (PR) 90/min, afebrile. Malar rash, photosensitive rash and mouth ulcers were present. Uterus was 30 weeks size relaxed. Fetal heart was 140 beats/min regular. On per vaginal examination, os was uneffaced and closed. Pedal edema was present. Patient is known case of SLE since 3 years and her ANA was positive. She was taking HCQ $200 \mathrm{mg}$ BD since 3 years and continued same in the pregnancy. She was taking heparin (low weight) and aspirin. She had preeclampsia since 26th week of gestation and was on labetalol $200 \mathrm{mg}$ since then. Doppler was suggestive of oligohydramnios and asymmetrical IUGR with fetoplacental-uteroplacental insufficiency. Patient was managed with antihypertensives and magnesium sulphate. She was taken for emergency caesarean section in view of fetal distress. A live female baby was born. Her post-partum period was uneventful.

\section{Case 3}

32 years G2P1L0, booked ANC with k/c/o SLE and lupus nephritis with hypothyroidism came for regular antenatal check-up at 13 weeks, 20 weeks and 26 weeks. On examination discoid lupus (Figure 3), nephritis and arthritis were present. She was managed with steroids, HCQ $300 \mathrm{mg}$, azathioprine, aspirin along with iron, calcium and folic acid. Her NT/NB scan, anomaly scan and dual marker were low risk.

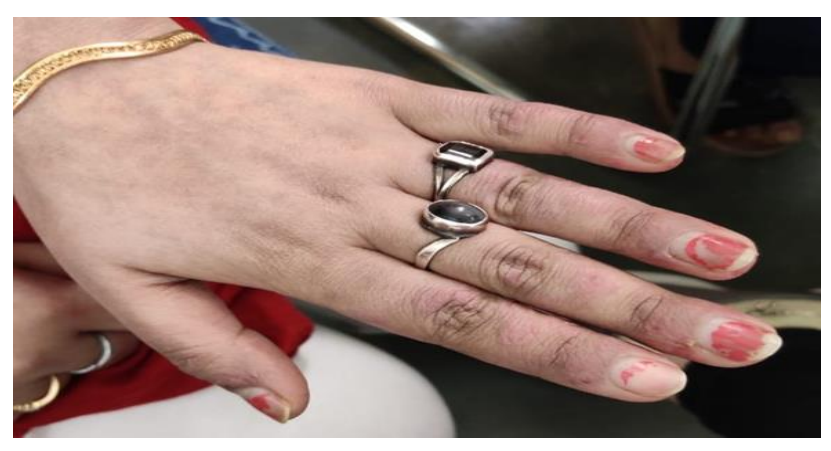

Figure 3: Discoid lupus.

She had previous term IUD due to unknown reasons that was delivered vaginally. Her anti-Ro and anti-la antibodies were positive. Patient lost follow up in $3^{\text {rd }}$ trimester. She came postnatally for regular check-up with an emergency caesarean section done at 35 weeks in view of IUGR and fetal distress. A male baby was born and her postpartum period remained uneventful.

\section{Case 4}

24 years old female presented to outpatient department (OPD) at 32 weeks pregnancy with oligohydramnios with positive APLA. There were pruritic urticarial papules and plaques with pyogenic granuloma on the feet. She had multiple cutaneous neurofibromas and café-au-lait spots 
(Figure 4). Ophthalmic examination was normal. Family history was positive for NF-1 (mother and brother). She was further investigated and diagnosed as NF type I. Patient was managed with hydration therapy and was put on aspirin. She went into preterm labour at 36 weeks of pregnancy and delivered a female baby by vaginal route. Delivery was uneventful. Baby was investigated for NF-1 and was found to be healthy.

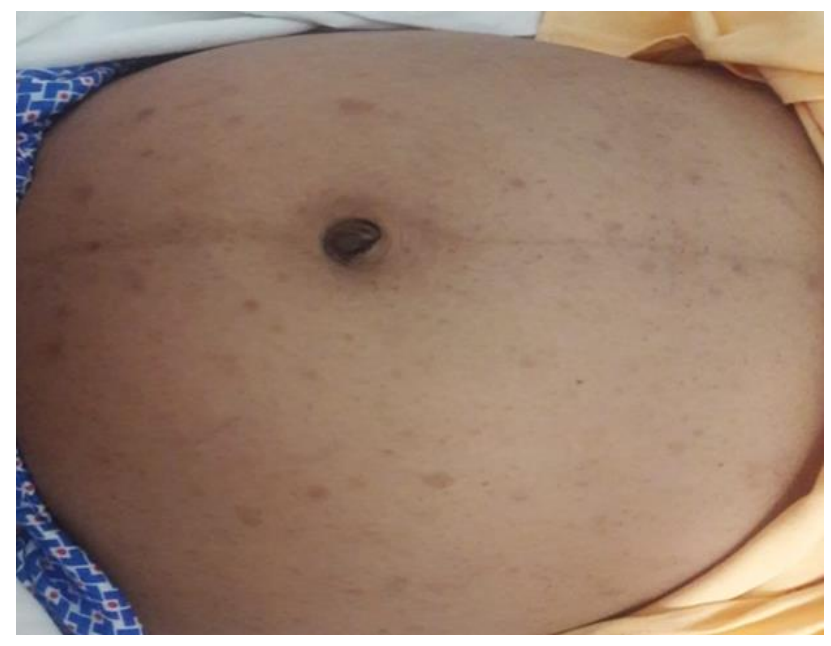

Figure 4: Cafe au lait spots.

\section{DISCUSSION}

Tuberous sclerosis is an autosomal dominant disorder with two genetic loci (tuberous sclerosis complex). The first gene TSC-1 is located on chromosome 9q34 and codes for protein hamartin. The second gene TSC-2 is located on chromosome $16 \mathrm{p} 13$ and codes for tuberin. Both of these are tumour suppressor genes regulating cellular growth and differentiation. The dysregulation in organogenesis results in development of various anomalies in multiple organs. $^{6}$

Tuberous sclerosis can affect all races, both sexes and it can present at any age. Its prevalence is around 1 in 6000 9000 individuals. $^{7}$ It can be inherited or can be a result of spontaneous genetic mutation. There is $50 \%$ chance of inheriting TSC in children if either parent has this condition. The characteristic feature of TS is its highly variable penetrance.

In infants, cardiac involvement and seizures are common presenting signs whereas skin and other organ involvement may suggest diagnosis in older individuals. ${ }^{8}$ In mother, neurological complications like intractable epilepsy, status epilepticus and subependymal giant cell astrocytoma (SEGA) are the most common cause of mortality and morbidity. Renal complications are the next most frequent cause. Less common are cardiac arrhythmias (which can present with sudden unexplained death), congestive heart failure and end-stage lung disease. Renal involvement appears to be the single most important prognostic factor in pregnancies with tuberous sclerosis. In tuberous sclerosis, a cardiac rhabdomyoma is the only sign that can be detected prenatally by ultrasound. ${ }^{9}$

Fetal cardiac rhabdomyomas are often benign and have a tendency to regress. It can occasionally lead to poor outcome and the need for surgery depending on the clinical presentation. Diagnosis is usually made on an obstetric ultrasonography between 21 to 30 weeks. ${ }^{10}$ In this case, it was diagnosed at 37 weeks by obstetric ultrasonography and fetal echocardiography. Our case had cardiac arrest for which CPR was done and she was revived. Later type 2 AV block was revealed in ECG and echocardiography revealed global hypokinesia of LV (ejection fraction 44\%).

Systemic lupus erythematosus (SLE) is one of the most common autoimmune disorders that affect women during childbearing years. SLE increases the risk of spontaneous abortion, intrauterine fetal death, preeclampsia, intrauterine growth retardation and preterm birth. The presence of ss-A and ss-B antibodies can lead to fetal heart block and neonatal lupus. The prognosis for both mother and child is best when SLE is quiescent for at least 6 months before the pregnancy with stable normal (or near normal) underlying renal function. SLE flares during pregnancy have been related to irreversible organ damage. However, whether pregnancy increases the risk of lupus flare is still unresolved, although the puerperium might be a period of particular high risk. Active lupus nephritis (LN) at conception and even in remission, confers a higher risk of flare during pregnancy. ${ }^{11}$

Pregnancy does not seem to endanger long-term renal function. Although, the higher the baseline creatinine, the greater the risk of deterioration. Lupus flares during pregnancy and postpartum are usually non-severe (i.e. articular, dermatological and mild haematological), However, severe flares with major organ involvement can occur. Given the multiple protective effects of hydroxychloroquine (HCQ), all women should be encouraged to continue to take HCQ throughout pregnancy and postpartum. ${ }^{12}$

Patients with SLE are at high risk of multiple medical (two- to four-fold increased risk of pre-eclampsia and thrombosis compared with the general population) and obstetric complications (preterm delivery 25\%; fetal growth restriction 6-35\%; and two- to six-fold risk of fetal loss compared with controls) during pregnancy, particularly in those with chronic hypertension, renal impairment and those taking high-dose oral steroids. Conversely, patients with only cutaneous lupus erythematosus or those with SLE in remission without major organ involvement appear to have pregnancy outcomes comparable to those of the healthy population. ${ }^{11}$

We observed preterm delivery and IUGR in both cases of SLE and preeclampsia in one. SLE didn't flare in both cases. 
Neurofibromatosis type 1 is one of the most common genetic disorders, caused by mutations of the NF-1 gene on chromosome 17 . This condition can either be inherited or occur de novo as a result of spontaneous mutations. ${ }^{5}$ About two thirds of the affected population with NF-1 have parents with NF-1 and inherit the disease from one of them. The risk for each child is estimated to be about $50 \%$. The remaining one third of the population develops this condition due to spontaneous mutations. It seems that most of the NF-1 mutations reported so far are unique. ${ }^{14}$ The clinical manifestations of NF-1 range from mild cutaneous lesions and axillary freckling to plexiform neurofibromas, optic gliomas, bony abnormalities, pseudoarthosis and malignancies. ${ }^{13}$

Published case reports demonstrate the association with intrauterine growth restriction, eclampsia, oligohydramnios, stillbirth, pregnancy-induced hypertension and preterm labor. ${ }^{14}$ Furthermore, pregnancy tends to increase the number and size of cutaneous neurofibromas with an apparent decrease in size subsequent to delivery. ${ }^{15}$ Some neurofibromas contain oestrogen receptors suggesting the existence of a correlation between the increase in number and size of neurofibromas with increase in serum oestrogen levels during pregnancy. ${ }^{16}$ Literature suggests that NF-1 may not be associated with significant obstetric complications and may have normal pregnancy outcome. ${ }^{15}$ Our case also showed that a normal obstetric outcome could be expected in pregnant women with NF-1.

\section{CONCLUSION}

Tuberous sclerosis is a rare genetic condition affecting multiple organs with hamartomas/tubers. It can adversely affect maternal and fetal outcome. Cardiac rhabdomyoma is the only sign that can be detected prenatally. In maternal tuberous sclerosis, fetal echocardiography is advisable at 22-24 weeks.

A pregnancy complicated by maternal or fetal tuberous sclerosis deserves regular antenatal follow up with necessary investigations. Genetic counseling is recommended for couples who have a family history of tuberous sclerosis. Prenatal diagnosis is available for families with a known gene mutation or history of this condition.

SLE is known to increase the risk of spontaneous miscarriage. It can also cause fetal growth restriction, sudden intrauterine death, pre-eclampsia and preterm delivery. Management of women with lupus nephritis can be difficult as the disease may mimic and overlap significantly with pre-eclampsia. Multidisciplinary management of pregnant women with SLE ensures optimum outcome of both mother and fetus. Clinicians have to make an accurate prenatal diagnosis, if possible.

The extreme variability of the phenotypic expression of the NF-1 gene makes it very difficult for NF-1 families to decide whether to have children or not, as molecular diagnosis cannot predict clinical expression of the disease. The psychological management of parents should therefore be very sensitive. Clinicians must discuss with parents the diagnostic possibilities and dilemmas during counseling and should keep in mind that a normal obstetric outcome could also occur in pregnant women with NF-1.

Funding: No funding sources

Conflict of interest: None declared

Ethical approval: Not required

\section{REFERENCES}

1. Yucesoy G, Ozkan S, Bodur H, Tan T, Calişkan E, Vural B, et al. Maternal and perinatal outcome in pregnancies complicated with hypertensive disorder of pregnancy: a seven year experience of a tertiary care center. Arch Gynecol Obstet. 2005;273:43-9.

2. Cauldwell M, Nelson-Piercy C. Maternal and fetal complications of systemic lupus erythematosus. Obstet Gynaecol. 2012;14:167-74.

3. Weiss SW, Goldblum JR. Enzinger and Weiss's Soft Tissue Tumors 4th Edition, St Luis, Mo: Mosby Inc, 2001.

4. Weismann A, Jakobi P, Zaidisk R. Neurofibromatosis and pregnancy. An update. J Reprod Med. 1993;38:890-6.

5. Sangwan N, Duhan N. Normal obstetric outcome in neurofibromatosis-1 complicating pregnancy. J Science. 2008;20:197-8.

6. Schwartz RA, Jozwiak S, Pedersen R, Bawle EV, Windle ML, Feld LG, et al. Genetics of Tuberous Sclerosis. Medscape. 2013.

7. Daroff RB, Fenichel GM, Jankovic J, Mazziotta JC. Bradley's Neurology in clinical practice. 6 thed Philadelphia: Elsevier. 2012;1508-14.

8. Gupta N, Singh N, Sarangi S, Dalmia S, Mittal S. Fetal cardiac rhabdomyoma with maternal tuberous sclerosis complicating pregnancy. Arch Gynecol Obstet. 2008;278(2):169-70.

9. Khanna PC, Godinho S, Pungavkar SA, Patkar DP. Ultrafast MRI in the prenatal diagnosis of Bourne ville's tuberous sclerosis. Neurol India. 2005;53(3):349-50.

10. Pipitone $\mathrm{S}$, Mongiovì $\mathrm{M}$, Grillo $\mathrm{R}$, Gagliano $\mathrm{S}$, Sperandeo V. Cardiac rhabdomyoma in intrauterine life: clinical features and natural history. A case series and review of published reports. Ital Heart J. 2002;3(1):48-52.

11. Ruiz-Irastorza G, Khamashta MA. Lupus and pregnancy: integrating clues from the bench and bedside. Eur J Clin Invest. 2011;41:672-8.

12. Ruiz-Irastorza G, Ramos-Casals M, Brito-Zeron P, Khamashta MA. Clinical efficacy and side effects of antimalarials in systemic lupus erythematosus: a systematic review. Ann Rheum Dis. 2010;69:20-8.

13. Strom CM, Strom S, Levine E, Ginsberg N, Barton J, Verlinsky Y. Obstetric outcomes in 102 pregnancies 
after preimplantation genetic diagnosis. Am J Obstet Gynecol. 2000;182:1629-32.

14. Kosec V, Márton I. Neurofibromatosis type 1 in pregnancy. Coll Antropol. 2006;30:247-9.

15. Dugoff L, Sujansky E. Neurofibromatosis type 1 and pregnancy. Am J Med Genet. 1996;66:7-10.

16. Leslie KK, White M, Sujansky E. Utilization of Polymerase Chain reaction (PCR) for Identification of Estrogen Receptors in Neurofibromas; Potential
Clinical Significance". National Neurofibromatosis Foundation. Annual Clinical Care Conference. 1992.

Cite this article as: Bedi M, Kaur S, Patil P, Kaur K, Kaur H, Kaur H. Diverse presentation of connective tissue disorders in pregnancy. Int J Reprod

Contracept Obstet Gynecol 2021;10:1681-5. 